In conclusion, Dr. Mascart points out that perhaps the most valuable result of the recent attack is the experience gained, and he emphasises the details in the programme which need greater attention, such as the closer observance of the precise prearranged hour, the correct orientation of the final drawing, the care which should be exercised in confirming the presence of a faint marking before showing it on the drawing, and so on. Finally, he gives, with full detail, descriptions of various objectives and eye-pieces, showing in each case, by means of diagrams, their action on rays of light.

Taken as a whole, this preliminary campaign appears to have provided very valuable results, and it is to be hoped that future similar organisations will be guided by the experience now gained.

\section{W. E. R.}

\section{THE NEW SCHOOL OF BOTANY, TRINITY COLLEGE, DUBIIN.}

THE science schools committee of Trinity College, Dublin, is to be congratulated on the completion of the second item in its scheme for the scientific development of Trinity College. This project, which was in the first instance originated by Prof. John Joly, F.R.S., and has since owed much to his activity and devotion, was inaugurated in $190 \mathrm{I}$ by a handsome subscription from the chancellor, the Earl of Rosse. At an early stage in the movement success was assured by a munificent gift from

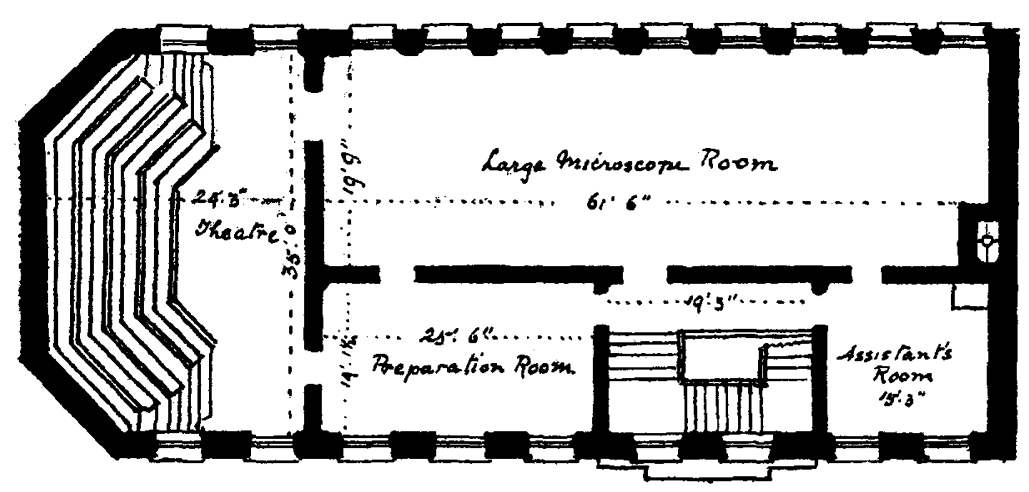

First Floor Plan.

Viscount Iveagh. According to the terms of this gift, Lord Iveagh undertook to build those departments for which the friends of Trinity College subscribed a capital sum requisite to yield the annual upkeep. Last year the School of Physics was completed, and at the beginning of the present session the School of Botany, the second department which has been benefited by this movement, was opened for work.

The School of Botany is a two-storied building of cut granite, situated in the east end of the college park, and lies east and west. This orientation gives a large number of windows facing north, with the best light for microscopic work. The western end, being octagonal, is occupied by a lecture theatre in the upper storey, on the ground floor by a very fine laboratory for general physiology. The northern side of the upper storey is occupied by a large microscope room, which can accommodate about sixty students working simultaneously. Through a large double door in the west end of this room arcess is obtained to the theatre. The auditorium in the latter is octagonal, so that every seat is close to the lecture table.

On the ground floor on the north side is a room for microscopic research, a library, professor's laboratory, and the laboratory for general physiology, which also forms the west end of the building. A solidly built greenhouse running out on the north side of the building is immediately connected with the physiological laboratory: on the south side of the latter opens a chemical laboratory In addition to the usual fittings in the physiological laboratory, there No. I994, voL. 77] is a large dark chamber, constructed like a fume cupboard, in which plants may be grown in complete darkness, or illuminated by light transmitted through special filters. The greenhouse has a separate heating sustem from the rest of the building, and a centrally placed case in it may be heated by a special radiator to a higher temperature than the rest of the greenhouse. Much thought has been given to the working out of the plan and fittings of this new school, and as it stands the School of Botany in Trinity College must rank with the best in the British Isles. The architect was Mr. Wm. C. Marshall, of London, who also designed the Botanical Laboratory in Cambridge.

\section{ON THE COLOURING MATTERS OF} FLOWERS.

THIRTY or forty years ago I devoted much attention to the colouring matters in plants, studying them with my newly invented spectrum microscope. I published a few papers on particular branches of the subject, but there are other very wide questions the importance of which I did not perceive until altered circumstances led me to devote my attention to work out at sea. Amongst other things studied was the variation in the colour of flowers, which is manifestly a very extensive subject, and for which I had only limited opportunity to obtain the requisite material, having to rely to a great extent on wild plants and flowers in my garden. Though the results are incomplete, they are probably characteristic; and it may be well to publish them, since it is now impossible for me to complete them, and what I did will at all events serve to show what might be done. The whole subject is very complex in more ways than one.

The colouring matters of plants mav be divided into two divisions, viz. those soluble in water but insoluble in carbon bisulphide or benzol, and those soluble in the latter reagents but not in water. Both are soluble in hydrous alcohol of the usual strength. Nearly all the blues and purples belong to the former, and most of the yellow and orange to the latter.

I found the best way of dealing with the flowers was to boil the petals or other portions in the usual hydrous alcohol, which dissolves both groups of pigments, and, after evaporating to dryness, to re-dissolve the constituents soluble in water, filter, and finally evaporate to dryness in a small saucer, in which, if kept fairly dry, the pigment will remain unchanged for a considerable time. Some pigments may be kept unchanged for a long time in a concentrated solution of lump sugar.

When re-dissolved in water many of the colouring matters soon become pale or nearly colourless, but recover their colour when evaporated to dryness. I never saw proof of this in living flowers, but it may occur when they die and fade. If there be any colour insoluble in water, it may be dissolved in carbon bisulphide, but this is seldom the case in blue, red, or purple flowers.

In those cases where the predominant colour is in soluble in water, it may be separated by agitating the alcoholic solution with carbon bisulphide, adding a little water. The bisulphide carries down the pigment in solution, which may then be evaporated to dryness in a small saucer and kept. When dissolved in alcohol or carbon bisulphide the colour fades more or less quickly, especially in the light, but if sealed up almost absolutely free from air, it will remain unchanged for many years, at all events in the dark.

Having, then, obtained the pigment in a fit state, the next thing is to examine it when in appropriate solution, either in its natural state or after the addition of a suitable reagent. To enter into full detail would make this paper far too long, but it seems desirable to give some particulars in order to show how the various pigment can be distinguished. Spcaking generally, this is by their 